\title{
Inverse dynamic problems for canonical systems and de Branges spaces
}

\author{
A. S. Mikhaylov ${ }^{1,2}$, V.S. Mikhaylov ${ }^{1,2}$ \\ ${ }^{1}$ St. Petersburg Department of V. A. Steklov Institute of Mathematics of the Russian Academy of Sciences, \\ 7 Fontanka, St. Petersburg, 191023 Russia \\ ${ }^{2}$ St. Petersburg State University, 7/9 Universitetskaya nab., St. Petersburg, 199034 Russia \\ mikhaylov@pdmi.ras.ru, vsmikhaylov@pdmi.ras.ru
}

DOI 10.17586/2220-8054-2018-9-2-215-224

\begin{abstract}
We show the equivalence of inverse problems for different dynamical systems and corresponding canonical systems. For canonical system with general Hamiltonian we outline the strategy of studying the dynamic inverse problem and procedure of construction of corresponding de Branges space.
\end{abstract}

Keywords: inverse problem, Boundary Control method, de Branges spaces, Schrödinger operator, Dirac system, Jacobi matrices, canonical systems.

Received: 8 January 2018

Revised: 19 January 2018

\section{Introduction}

This is an accompanying paper to [1], in which the authors have shown the relationship between the de Branges method and the Boundary Control (BC) method on a basis of three dynamical systems: wave equation with a potential on a half-line, Dirac system on a half-line and dynamical system with discrete time for semiinfinite discrete Schrödinger operator. For each system, they constructed the related de Branges space using natural dynamic objects and operators, used in the $\mathrm{BC}$ method. In the present note, we will show the equivalence of dynamic inverse problems (IP) for different dynamical systems (wave equation, Dirac system, Jacobi matrices), and IPs for equivalent canonical systems. We note that every original system will be equivalent to canonical system with different dynamics (the dependence on $t$ is given by one of the following operators: $\frac{d^{2}}{d t^{2}}, i \frac{d}{d t}, \partial_{t}$, where $\partial_{t}$ is a difference operator).

Let $H \in L_{1, l o c}\left(0, L ; \mathbb{R}^{2 \times 2}\right)$ be a locally summable on $(0, L), L \leq \infty$ matrix-valued function $H \geq 0$, called Hamiltonian, $J:=\left(\begin{array}{cc}0 & 1 \\ -1 & 0\end{array}\right)$, vector $Y=\left(\begin{array}{l}Y_{1} \\ Y_{2}\end{array}\right)$. We choose the "proper" dynamics and fix the general dynamical canonical system, the initial boundary value problem (IBVP) of which will be the subject of our interest:

$$
i H \frac{d Y}{d t}-J \frac{d Y}{d x}=0, \quad x \geq 0, t \geq 0 .
$$

For such a system we set up an IP and outline the strategy of solving it by the BC method, provided the Hamiltonian is smooth and strictly positive. We also provide a method of construction of the de Branges space for such a Hamiltonian in natural dynamic terms following [1].

In the second section, we expose all necessary information on de Branges spaces and canonical systems following [2] and [3]. In the third section, we deal with dynamical systems for Schrödinger operator on a half-line, wave equation on a half-line, Dirac operator on a half-line and a semi-infinite Jacobi matrices. We formulate dynamic IP for each system, then we transform IBVP for each system to the IBVP for certain canonical system, formulate IP for canonical system, and show that it is equivalent the original ones.

In the fourth section, we will show that one specific choice of dynamics give a finite speed of wave propagation in a canonical system, provided the Hamiltonian is smooth and strictly positive. We note that the finiteness of the wave propagation is important: initially the BC method was developed and applied in the case of multidimensional wave equation [4,5] on a bounded manifold, but later on the BC method was successfully applied to parabolic and Schrödinger equations (where the speed is infinite) as well [6-8]. We provide algorithms of solving dynamic IP and construction of de Branges space for such a Hamiltonian. Based on these results, we formulate the hypothesis for constructing the de Branges space for general Hamiltonian by the dynamic method. 


\section{2. de Branges spaces}

Here, we provide the information on de Branges spaces in accordance with $[2,3]$. The entire function $E$ : $\mathbb{C} \mapsto \mathbb{C}$ is called a Hermite-Biehler function if $|E(z)|>|E(\bar{z})|$ for $z \in \mathbb{C}_{+}$. We use the notation $F^{\#}(z)=\overline{F(\bar{z})}$. The Hardy space $H_{2}$ is defined by: $f \in H_{2}$ if $f$ is holomorphic in $\mathbb{C}^{+}$and $\sup _{y>0} \int_{-\infty}^{\infty}|f(x+i y)|^{2} d x<\infty$. Then the de Branges space $B(E)$ consists of entire functions such that:

$$
B(E):=\left\{F: \mathbb{C} \mapsto \mathbb{C}, F \text { entire, } \int_{\mathbb{R}}\left|\frac{F(\lambda)}{E(\lambda)}\right|^{2} d \lambda<\infty, \frac{F}{E}, \frac{F^{\#}}{E} \in H_{2}\right\} .
$$

The space $B(E)$ with the scalar product:

$$
[F, G]_{B(E)}=\frac{1}{\pi} \int_{\mathbb{R}} \overline{F(\lambda)} G(\lambda) \frac{d \lambda}{|E(\lambda)|^{2}},
$$

is a Hilbert space. For any $z \in \mathbb{C}$ the reproducing kernel is introduced by the relation

$$
J_{z}(\xi):=\frac{\overline{E(z)} E(\xi)-E(\bar{z}) \overline{E(\bar{\xi})}}{2 i(\bar{z}-\xi)} .
$$

Then

$$
F(z)=\left[J_{z}, F\right]_{B(E)}=\frac{1}{\pi} \int_{\mathbb{R}} \overline{J_{z}(\lambda)} F(\lambda) \frac{d \lambda}{|E(\lambda)|^{2}} .
$$

We observe that a Hermite-Biehler function $E(\lambda)$ defines $J_{z}$ by (1). The converse is also true [9,10]: a Hilbert space of analytic functions with reproducing kernel is a de Branges space (provided some nonrestrictive conditions on the set of function and on the norm hold true).

Let $H \in L_{1, l o c}\left(0, L ; R^{2 \times 2}\right)$ be a Hamiltonian and the vector $Y=\left(\begin{array}{c}Y_{1} \\ Y_{2}\end{array}\right)$ be solution to the following Cauchy problem:

$$
\begin{aligned}
-J \frac{d Y}{d x} & =\lambda H Y, \\
Y(0) & =C,
\end{aligned}
$$

for $C \in \mathbb{R}^{2}, C \neq 0$. Without loss of generality, it is assumed that $\operatorname{tr} H(x)=1$. Then, the function $E_{x}(\lambda)=$ $Y_{1}(x, \lambda)+i Y_{2}(x, \lambda)$ is a Hermite-Biehler function $\left(E_{L}(\lambda)\right.$ makes sense if $\left.L<\infty\right)$, it is called de Branges function of the system (2) since one can construct de Branges space based on this function. On the other hand, $E_{L}$ serves as an inverse spectral data for the canonical system (2). The solution to (2) and $Y(0)=(1,0)^{T}$ is denoted by $\Theta(x, \lambda)$. The main result of the theory [3,9] says that the opposite is also true: every Hermite-Biehler function satisfying some condition comes from some canonical system.

\section{Dynamical canonical systems for wave equation, Dirac system and Jacobi system with discrete time}

In this section, we use some ideas from [3] to rewrite IBVPs for different dynamical systems as IBVPs for canonical dynamical systems. Everywhere below, $T>0$ is fixed.

\subsection{Wave equation with a potential on a half-line}

For a potential $q \in L_{1, \text { loc }}\left(\mathbb{R}_{+}\right)$, we consider the IBVP for the $1 \mathrm{~d}$ wave equation on a half-line:

$$
\left\{\begin{array}{l}
u_{t t}(x, t)-u_{x x}(x, t)+q(x) u(x, t)=0, \quad x \geq 0, t \geq 0, \\
u(x, 0)=u_{t}(x, 0)=0, u(0, t)=f(t) .
\end{array}\right.
$$

Here, $f$ is an arbitrary $L_{l o c}^{2}\left(\mathbb{R}_{+}\right)$function referred to as a boundary control. The response operator $R_{q}^{T}$ : $L_{2}(0, T) \mapsto L_{2}(0, T)$ with the domain $\mathcal{D}=C_{0}^{\infty}(0, T)$ is introduced by $\left(R_{q}^{T} f\right)(t):=u_{x}^{f}(0, t)$, it plays a role of a dynamic inverse data [11-13]. The IP is to recover q on $(0, T)$ from $R_{q}^{2 T}$.

We consider the solutions $y_{1,2}$ to following Cauchy problems:

$$
\left\{\begin{array}{l}
-y_{1,2}^{\prime \prime}(x)+q(x) y_{1,2}(x)=0, \quad x \geq 0 \\
y_{1}(0)=1, y_{1}^{\prime}(0)=0, y_{2}(0)=0, y_{2}^{\prime}(0)=1
\end{array}\right.
$$


and look for the solution to (3) in the form:

$$
u^{f}(x, t)=c^{1}(x, t) y_{1}(x)+c^{2}(x, t) y_{2}(x) .
$$

Plugging this representation to (3) yields:

$$
\begin{array}{r}
c_{t t}^{1} y_{1}+c_{t t}^{2} y_{2}=-q c^{1} y_{1}-q c^{2} y_{2}+c_{x x}^{1} y_{1}+2 c_{x}^{1} y_{1}^{\prime}+c_{1} y_{1}^{\prime \prime}+c_{x x}^{2} y_{2}+2 c_{x}^{2} y_{2}^{\prime}+c_{2} y^{\prime \prime} \\
=\left(c_{x}^{1} y_{1}+c_{x}^{2} y_{2}\right)_{x}+c_{x}^{1} y_{1}^{\prime}+c_{x}^{2} y_{2}^{\prime} .
\end{array}
$$

If we demand the equality $c_{x}^{1} y_{1}+c_{x}^{2} y_{2}=0$, then unknown $c^{1,2}$ satisfies the following system:

$$
\left\{\begin{array}{l}
c_{t t}^{1} y_{1}+c_{t t}^{2} y_{2}=c_{x}^{1} y_{1}^{\prime}+c_{x}^{2} y_{2}^{\prime} \\
c_{x}^{1} y_{1}+c_{x}^{2} y_{2}=0 .
\end{array}\right.
$$

We note that due to the boundary conditions in (4) and (6), we have that:

$$
u_{x}^{f}(0, t)=c_{x}^{1}(0, t) y_{1}(0)+c^{1}(0, t) y_{1}^{\prime}(0)+c_{x}^{2}(0, t) y_{2}(0)+c^{2}(0, t) y_{2}^{\prime}(0)=c^{2}(0, t) .
$$

On expressing $c_{x}^{1,2}$ from (6), and bearing in mind the equality $\operatorname{det}\left(\begin{array}{ll}y_{1} & y_{2} \\ y_{1}^{\prime} & y_{2}^{\prime}\end{array}\right)=1$, we obtain that:

$$
\left\{\begin{array}{l}
c_{x}^{1}=-c_{t t}^{1} y_{1} y_{2}-c_{t t}^{2} y_{2}^{2} \\
c_{x}^{2}=c_{t t}^{1} y_{1}^{2}+c_{t t}^{2} y_{1} y_{2}
\end{array}\right.
$$

On introducing the notations $C=\left(\begin{array}{l}c^{1} \\ c^{2}\end{array}\right), J=\left(\begin{array}{cc}0 & 1 \\ -1 & 0\end{array}\right), H=\left(\begin{array}{cc}y_{1}^{2} & y_{1} y_{2} \\ y_{1} y_{2} & y_{2}^{2}\end{array}\right)$ and counting the initial and boundary conditions on $u^{f}$ at $t=0$ and at $x=0$, we obtain that $C$ satisfies the following IBVP:

$$
\begin{cases}H C_{t t}-J C_{x}=0, & x \geq 0, t \geq 0 \\ C(x, 0)=0, C_{t}(x, 0)=0, & x \geq 0 \\ c^{1}(0, t)=f(t), & t \geq 0\end{cases}
$$

The response operator $\widetilde{R}_{q}^{T}: L_{2}(0, T) \mapsto L_{2}(0, T)$ for $(7)$ is introduced by the equality $\left(\widetilde{R}_{s}^{T} f\right)(t):=c^{2}(0, t)$. On the other hand, using (5) and second line in (6), we have that:

$$
\left(R_{q}^{T} f\right)(t):=u_{x}^{f}(0, t)=c_{x}^{1}(0, t) y_{1}(0)+c^{2}(0, t) y_{2}^{\prime}(0)=c^{2}(0, t)=\left(\widetilde{R}_{s}^{T} f\right)(t) .
$$

So we can see that IPs for (3) and for (7) are equivalent.

\subsection{Wave equation on a half-line}

For a smooth positive density $\rho \in C^{2}\left(\mathbb{R}_{+}\right), \rho(x) \geq \delta>0$, we consider the IBVP for a wave equation on a half-line:

$$
\left\{\begin{array}{l}
\rho(x) u_{t t}(x, t)-u_{x x}(x, t)=0, \quad x \geq 0, t \geq 0 \\
u(x, 0)=u_{t}(x, 0)=0, u(0, t)=f(t) .
\end{array}\right.
$$

Where the function $f \in L_{\text {loc }}^{2}\left(\mathbb{R}_{+}, \mathbb{C}\right)$ is interpreted as a boundary control. The response operator $R_{\rho}^{T}: L_{2}(0, T) \mapsto$ $L_{2}(0, T)$ with the domain $\mathcal{D}=C_{0}^{\infty}(0, T)$ is defined by $R_{\rho}^{T} f:=u_{x}^{f}(0, t)$. We introduce the eikonal $\tau(x):=$ $\int_{0}^{x} \rho^{\frac{1}{2}}(s) d s$, from physical point of view, it is a time at which a wave initiated at $x=0$ fills the segment $(0, x)$, let $\Omega^{l}=\{x>0 \mid \tau(x)<l\}$. Then, the natural set up of IP is to recover $\left.\rho(x)\right|_{\Omega^{T}}$ from $R_{\rho}^{2 T}$, see [14].

We introduce the new function:

$$
C(x, t)=\left(\begin{array}{c}
c^{1} \\
c^{2}
\end{array}\right):=\left(\begin{array}{c}
u_{t} \\
i u_{x}
\end{array}\right)
$$

and a Hamiltonian $H:=\left(\begin{array}{cc}\rho(x) & 0 \\ 0 & 1\end{array}\right)$. Then it is easy to see that $Y$ satisfies the canonical system:

$$
\begin{cases}i H C_{t}-J C_{x}=0, & x \geq 0, t \geq 0, \\ C(x, 0)=0, & x \geq 0, \\ c^{1}(0, t)=g(t):=f^{\prime}(t), & t \geq 0 .\end{cases}
$$

The response operator $\widetilde{R}_{\rho}^{T}: L_{2}(0, T) \mapsto L_{2}(0, T)$ for (9) with the domain $\mathcal{D}=C_{0}^{\infty}(0, T)$ is introduced by $\left(\widetilde{R}_{s}^{T} g\right)(t):=c^{2}(0, t)$. We can see that IPs for (8) and for (9) are equivalent. 


\subsection{Dirac system on a half-line}

With a matrix potential $V=\left(\begin{array}{cc}p & q \\ q & -p\end{array}\right), p, q \in C_{\text {loc }}^{1}\left(\mathbb{R}_{+}\right)$, vector $u=\left(\begin{array}{l}u_{1} \\ u_{2}\end{array}\right)$ we associate the IBVP for a Dirac system:

$$
\begin{cases}i u_{t}+J u_{x}+V u=0, & x \geq 0, t \geq 0, \\ \left.u\right|_{t=0}=0, & x \geq 0, \\ \left.u_{1}\right|_{x=0}=f, & t \geq 0,\end{cases}
$$

Here $f$ is an arbitrary $L_{\text {loc }}^{2}\left(\mathbb{R}_{+}, \mathbb{C}\right)$ function referred to as a boundary control. The response operator $R_{D}^{T}$ : $L_{2}(0, T) \mapsto L_{2}(0, T)$ with the domain $\mathcal{D}=C_{0}^{\infty}(0, T)$ is introduced by $\left(R_{D}^{T} f\right)(t):=u_{2}(0, t)$, it plays a role of a dynamic inverse data. The IP is to recover $V$ on $(0, T)$ from $R_{D}^{2 T}$, see [15].

Let $Y^{1,2}$ be solutions to the following Cauchy problems:

$$
\left\{\begin{array}{l}
J Y_{x}^{1,2}+V Y^{1,2}=0 \\
Y_{1}^{1}(0)=1, \quad Y_{2}^{1}(0)=0, \quad Y_{1}^{2}(0)=0, \quad Y_{2}^{2}(0)=1 .
\end{array}\right.
$$

We will look for the solution to (10) in the form:

$$
u(x, t)=c^{1}(x, t) Y^{1}(x)+c^{2}(x, t) Y^{2}(x) .
$$

Plugging this representation in (10) yields:

$$
\begin{array}{r}
i\left(c_{t}^{1} Y^{1}+c_{t}^{2} Y^{2}\right)+c_{x}^{1} J Y^{1}+c_{x}^{2} J Y^{2}+c_{1} J Y_{x}^{1}+c_{2} J Y_{x}^{2}+c_{1} V Y^{1}+c_{2} V Y^{2} \\
=i\left(c_{t}^{1} Y^{1}+c_{t}^{2} Y^{2}\right)+J\left(c_{x}^{1} Y^{1}+c_{x}^{2} Y^{2}\right)=0
\end{array}
$$

on introducing $C=\left(\begin{array}{l}c^{1} \\ c^{2}\end{array}\right)$, we see that the above equality is equivalent to:

$$
i\left(\begin{array}{cc}
Y_{1}^{1} & Y_{1}^{2} \\
Y_{2}^{1} & Y_{2}^{2}
\end{array}\right) C_{t}+J\left(\begin{array}{cc}
Y_{1}^{1} & Y_{1}^{2} \\
Y_{2}^{1} & Y_{2}^{2}
\end{array}\right) C_{x}=0
$$

We introduce the notation: $A=\left(\begin{array}{cc}Y_{1}^{1} & Y_{1}^{2} \\ Y_{2}^{1} & Y_{2}^{2}\end{array}\right), B=J A J$. Then the above system is equivalent to:

$$
i A C_{t}-B J C_{x}=0,
$$

on multiplying it by $B^{-1}$ and introducing the Hamiltonian by $H=B^{-1} A$, we obtain:

$$
i H C_{t}-J C_{x}=0 .
$$

Counting that $\operatorname{det} B=\operatorname{det} A=1$, we evaluate:

$$
H=B^{-1} A=\left(\begin{array}{cc}
Y^{1} Y^{1} & Y^{1} Y^{2} \\
Y^{1} Y^{2} & Y^{2} Y^{2}
\end{array}\right)
$$

Bearing in mind the initial and boundary conditions in (10), we see that $C$ satisfies the following IBVP:

$$
\begin{cases}i H C_{t}-J C_{x}=0, & x \geq 0, t \geq 0, \\ C(x, 0)=0, & x \geq 0, \\ c^{1}(0, t)=f(t), & t \geq 0 .\end{cases}
$$

The response operator $\widetilde{R}_{D}^{T}: L_{2}(0, T) \mapsto L_{2}(0, T)$ for $(12)$ is introduced by $\left(\widetilde{R}_{D}^{T} f\right)(t):=c^{2}(0, t)$. The representation (11) implies that IPs for (10) and for (12) are equivalent.

\subsection{Semi-infinite Jacobi matrices}

Let $0=b_{0}<b_{1}<b_{2}<\ldots<b_{n}<\ldots$ be a partition of $[0,+\infty)$. We introduce the notations: $\Delta_{j}:=$ $\left(b_{j-1}, b_{j}\right), l_{j}=\left|\Delta_{j}\right|=b_{j}-b_{j-1}$. Let for each $j$ we define $e_{j} \in \mathbb{R}^{2},\left|e_{j}\right|=1, e_{j} \neq \pm e_{j \pm 1}$, and $e_{j}(x)=e_{j}$, $x \in \Delta_{j}$. We define a Hamiltonian $H$ :

$$
H(x) f(x)=\left(f(x), e_{j}(x)\right) e_{j}(x)=\left(\begin{array}{cc}
e_{1 j}^{2}(x) & e_{1 j}(x) e_{2 j}(x) \\
e_{1 j}(x) e_{2 j}(x) & e_{2 j}^{2}(x)
\end{array}\right)\left(\begin{array}{l}
f^{1}(x) \\
f^{2}(x)
\end{array}\right)
$$

Consider functions of the type (i.e. functions from the domain of operator, corresponding to such a Hamiltonian, see $[R R])$ :

$$
f(x)=\left(\begin{array}{l}
f^{1}(x) \\
f^{2}(x)
\end{array}\right)=f_{j} e_{j}(x)+\xi_{j}(x) e_{j}^{\perp}(x), \quad x \in \Delta_{j}, f_{j} \in \mathbb{R}, \quad e_{j}^{\perp}=J e_{j},
$$


and note that $\left(f, e_{j}\right)=f_{j}$. For such a Hamiltonian $H$ we study the equation:

$$
J f^{\prime}=H g,
$$

where the function $g$ has a form (13), $g=g_{j} e_{j}(x)+\eta_{j}(x) e_{j}^{\perp}(x), x \in \Delta_{j}$. The equality in (14) implies that

$$
\xi_{j}^{\prime}(x) J e_{j}^{\perp}(x)=g_{j} e_{j}(x), \quad x \in \Delta_{j},
$$

which yields the following expression for $\xi_{j}(x)$ for some $s_{j}$ :

$$
\xi_{j}(x)=s_{j}+g_{j}\left(b_{j}-x\right), \quad x \in \Delta_{j} .
$$

We use the continuity condition at $x=b_{j-1}$ to obtain:

$$
f_{j-1} e_{j-1}+s_{j-1} e_{j-1}^{\perp}=f_{j} e_{j}+\left(s_{j}+g_{j} l_{j}\right) e_{j}^{\perp} .
$$

Multiplying the above equality by $e_{j}$ we get:

$$
s_{j-1}=\frac{1}{\left(e_{j}, e_{j-1}^{\perp}\right)}\left(f_{j}-f_{j-1}\left(e_{j}, e_{j-1}^{\perp}\right)\right),
$$

and multiplying by $e_{j-1}$ we obtain:

$$
f_{j-1}=f_{j}\left(e_{j}, e_{j-1}\right)+\left(s_{j}+g_{j} l_{j}\right)\left(e_{j}^{\perp}, e_{j-1}\right) .
$$

Using (16), (17) we can express $g_{j}$ via $f_{j-1}, f_{j}, f_{j+1}$ :

$$
g_{j} l_{j}=\frac{1}{\left(e_{j}, e_{j-1}^{\perp}\right)} f_{j-1}+\left(\frac{\left(e_{j+1}, e_{j}\right)}{\left(e_{j+1}, e_{j}^{\perp}\right)}-\frac{\left(e_{j}, e_{j-1}\right)}{\left(e_{j}^{\perp}, e_{j-1}\right)}\right) f_{j}-\frac{1}{\left(e_{j}^{\perp}, e_{j+1}\right)} f_{j+1} .
$$

Making the substitution:

from (18) we obtain the relation:

$$
u_{j}=g_{j} \sqrt{l_{j}}, \quad v_{j}=f_{j} \sqrt{l_{j}},
$$

$$
u_{j}=\frac{1}{\left(e_{j}, e_{j-1}^{\perp}\right) \sqrt{l_{j-1} l_{j}}} v_{j-1}+\frac{1}{l_{j}}\left(\frac{\left(e_{j+1}, e_{j}\right)}{\left(e_{j+1}, e_{j}^{\perp}\right)}-\frac{\left(e_{j}, e_{j-1}\right)}{\left(e_{j}^{\perp}, e_{j-1}\right)}\right) v_{j}-\frac{1}{\left(e_{j}^{\perp}, e_{j+1}\right) \sqrt{l_{j} l_{j+1}}} v_{j+1} .
$$

On introducing the notations:

$$
\begin{array}{r}
\rho_{j}=\frac{-1}{\left(e_{j+1}, e_{j}^{\perp}\right) \sqrt{l_{j} l_{j+1}}}, \quad j \geq 1, \\
q_{j}=\frac{1}{l_{j}}\left(\frac{\left(e_{j}, e_{j+1}\right)}{\left(e_{j}^{\perp}, e_{j+1}\right)}-\frac{\left(e_{j}, e_{j-1}\right)}{\left(e_{j}^{\perp}, e_{j-1}\right)}\right), \quad j \geq 2,
\end{array}
$$

we can rewrite (20) in a form:

$$
u_{j}=\rho_{j-1} v_{j-1}+q_{j} v_{j}+\rho_{j} v_{j+1}, \quad j \geq 2,
$$

and $q_{1}$ is found from the condition at zero. So finally we obtain the following result: if $f$ and $g$ having representation (13) are connected by (14), then $u$ and $v$ defined by (19) satisfy:

$$
A v=u, \quad A=\left(\begin{array}{ccccc}
q_{1} & \rho_{1} & 0 & 0 & 0 \\
\rho_{1} & q_{2} & \rho_{2} & 0 & 0 \\
0 & \rho_{2} & q_{3} & \rho_{3} & 0 \\
0 & 0 & \cdot & \cdot & .
\end{array}\right) .
$$

We can introduce the dependence on (continuous) time $t$ : let $f(x, t), g(x, t)$ have form:

$$
\begin{gathered}
f(x, t)=f_{j}(t) e_{j}(x)+\xi(x, t) e_{j}^{\perp}(x), \quad x \in \Delta_{j}, \\
g(x, t)=g_{j}(t) e_{j}(x)+\eta(x, t) e_{j}^{\perp}(x), \quad x \in \Delta_{j},
\end{gathered}
$$

then if $g(x, t)=i f_{t}(x, t)$, then $f$ solves:

$$
J f_{x}=i H f_{t}
$$

On the other hand (19) implies the relationship $u_{j}(t)=i v_{j_{t}}(t)$, which yields that $v$ solves $i v_{t}-A v=0$. Adding initial and boundary conditions gives well-posed IBVP for dynamical system with continuous time governed by Jacobi matrix:

$$
\begin{cases}i v_{t}-A v=0, & x \geq 0, t \geq 0 \\ v_{n}(0)=0, & n \geq 1 \\ v_{1}(t)=h(t), & t \geq 0\end{cases}
$$


The response operator $R_{J}^{T}: L_{2}(0, T) \mapsto L_{2}(0, T)$ with the domain $D=C_{0}^{\infty}(0, T)$ for this system is introduced by the rule $\left(R_{J}^{T} h\right)(t):=v_{2}(t)$. On the other hand, IBVP (21) is equivalent to (we assume that $e_{1}=(1,0)^{T}$ ):

$$
\begin{cases}i H f_{t}-J f_{x}=0, & x \geq 0, t \geq 0, \\ f(x, 0)=0, & x \geq 0 \\ f^{1}(0, t)=j(t):=\frac{h(t)}{\sqrt{l_{1}}}, & t \geq 0 .\end{cases}
$$

For the system (22), the response operator $\widetilde{R}_{J}^{T}: L_{2}(0, T) \mapsto L_{2}(0, T)$ with the domain $D=C_{0}^{\infty}(0, T)$ is introduced by the rule $\left(R_{J}^{T} h\right)(t):=f^{2}(0, t)$. Note that by (13), $f^{2}(0, t)=\xi_{1}(0, t)$. From (15), the relationship $g(x, t)=i f_{t}(x, t)$ and $(16)$, we have that:

$$
\begin{array}{r}
\left(R_{J}^{T} h\right)(t)=s_{1}(t)+g_{1}(t) l_{1}=\frac{f_{2}(t)}{\left(e_{2}, e_{1}^{\perp}\right)}-f_{1}(t)+i f^{1}(0, t) l_{1} \\
=\frac{f_{2}(t)}{\left(e_{2}, e_{1}^{\perp}\right)}-\frac{h(t)}{\sqrt{l_{1}}}+i h(t) \sqrt{l_{1}}=-\rho_{1} v_{2}(t) \sqrt{l_{1}}-h(t)\left(\frac{1}{\sqrt{l_{1}}}-i \sqrt{l_{1}}\right) .
\end{array}
$$

So IP for (21) and (22) from corresponding response operators are equivalent. We note that we can introduce the different type of continuous dynamics for Jacobi matrices (for example the dynamics of the type $\frac{d}{d t^{2}}$ was considered in [16]).

We can also introduce the dependence on the discrete time $t \in \mathbb{N}$ by letting $f_{t}(x), g_{t}(x)$ have form:

$$
\begin{aligned}
& f_{t}(x)=f_{j, t} e_{j}(x)+\xi_{t}(x) e_{j}^{\perp}(x), \quad x \in \Delta_{j}, t \in \mathbb{N}, \\
& g_{t}(x)=g_{j, t} e_{j}(x)+\eta_{t}(x) e_{j}^{\perp}(x), \quad x \in \Delta_{j}, t \in \mathbb{N} .
\end{aligned}
$$

If $f, g$ are related by $g_{t}(x)=f_{t}(x)+f_{t-1}(x)=: \partial_{t} f(x)$, then counting (14), $f$ solves:

$$
J f_{x}=H \partial_{t} f \text {. }
$$

The equality (19) implies $u_{j}=\partial_{t} v_{j}$, which yields that $v$ satisfies $\partial_{t} v_{\cdot, t}-A v_{\cdot, t}=0$. Adding initial and boundary conditions gives the following IBVP:

$$
\begin{cases}\partial_{t} v_{\cdot, t}-A v_{\cdot, t}=0, & t \in \mathbb{N} \\ v_{n, 1}=v_{n, 0}(0)=0, & n \geq 1 \\ v_{1, t}=h_{t}, & t \in \mathbb{N}\end{cases}
$$

where $h_{t} \in l_{2}$ is referred to as a boundary control. The response operator $R_{J, d}^{T}$ with the domain $D=\mathrm{R}^{T}$ for this system is introduced by $R_{J, d}^{T}: \mathbb{R}^{T} \mapsto \mathbb{R}^{T},\left(R_{J, d}^{T} h\right)_{t}=v_{2, t}, t=1 \ldots, T$. The forward and inverse problem was studied in $[17,18]$. The IBVP (23) is equivalent to, which is equivalent to the following IBVP for a canonical system:

$$
\begin{cases}H \partial_{t} f-J f_{x}=0, & x \geq 0, t \in \mathbb{N} \\ f_{0}(x)=0, & x \geq 0 \\ f_{t}^{1}(0)=j_{t}:=\frac{h_{t}}{\sqrt{l_{1}}}, & t \in \mathbb{N}\end{cases}
$$

For the system (24) the response operator $\widetilde{R}_{J, d}^{T}: l_{2} \mapsto l_{2}$ is introduced by the rule $\left(R_{J}^{T} j\right)(t):=f_{t}^{2}(0)$. By (13), $f_{t}^{2}(0)=\xi_{1 t}(0)$, from (15), the relationship $g_{t}(x)=\partial_{t} f(x)$ and (16), we have that:

$$
\begin{array}{r}
\left(R_{J, d}^{T} h\right)_{t}=s_{1 t}+g_{1 t} l_{1}=\frac{f_{2 t}}{\left(e_{2}, e_{1}^{\perp}\right)}-f_{1 t}+i f_{t}^{1}(0) l_{1} \\
=\frac{f_{2 t}}{\left(e_{2}, e_{1}^{\perp}\right)}-\frac{h_{t}}{\sqrt{l_{1}}}+i h_{t} \sqrt{l_{1}}=-\rho_{1} v_{2, t} \sqrt{l_{1}}-h_{t}\left(\frac{1}{\sqrt{l_{1}}}-i \sqrt{l_{1}}\right) .
\end{array}
$$

So, IP for (23) and (24) from corresponding response operators are equivalent.

We see that different dynamic systems after transformations come to dynamical canonical systems with different dynamics $\left(i \frac{d}{d t}, \frac{d}{d t^{2}}\right.$, and even discrete one $\left.\partial_{t}\right)$.

We will investigate the dynamics given by $i \frac{d}{d t}$, the canonical system with this dynamics possess property of finite speed of wave propagation. 


\section{Canonical systems with smooth strictly positive Hamiltonian}

We consider the IBVP for a canonical system. Assuming that the Hamiltonian satisfies conditions: $H=H^{*} \in$ $C^{2}\left(0, T ; \mathbb{R}^{2 \times 2}\right), H \geq \delta>0, \operatorname{tr} H=1$, we set $Y^{f}=\left(\begin{array}{l}y^{1} \\ y^{2}\end{array}\right)$ to be a solution to:

$$
\begin{cases}i H \frac{d}{d t} Y-J \frac{d}{d x} Y=0, & x \geq 0, t \geq 0 \\ Y(x, 0)=0, & x \geq 0 \\ y^{1}(0, t)=f(t), & t \geq 0 .\end{cases}
$$

Where the boundary control $f \in \mathcal{F}^{T}:=L_{2}(0, T ; \mathbb{C})$. The response operator $R^{T}: \mathcal{F}^{T} \mapsto \mathcal{F}^{T}$ is introduced as $\left(R^{T} f\right)(t):=y_{2}^{f}(0, t)$. The inverse problem we will be dealing with consists in a recovering $H(x)$, on an interval $(0, l)$ for some $l>0$ from given $R^{2 T}$.

\subsection{One-velocity wave system}

We rewrite (25): differentiate the first line in (25) w.r.t. $t$ and use equation to get:

$$
H Y_{t t}+J H^{-1} J Y_{x x}+J H_{x}^{-1} J Y_{x}=0
$$

which is equivalent to the equation:

$$
H Y_{t t}-\frac{1}{\operatorname{det} H} H Y_{x x}+J H_{x}^{-1} J Y_{x}=0
$$

Counting the initial and boundary condition, we obtain that $Y$ satisfies the following IBVP for one-velocity system:

$$
\begin{cases}\operatorname{det} H Y_{t t}-Y_{x x}+\operatorname{det} H H^{-1} J H_{x}^{-1} J Y_{x}=0, & x \geq 0, t \geq 0 \\
Y(x, 0)=Y_{t}(x, 0)=0, & x \geq 0, \\
\left(\begin{array}{l}
y^{1}(0, t) \\
y^{2}(0, t)
\end{array}\right)=G(t):=\left(\begin{array}{c}
f(t) \\
(R f)(t)
\end{array}\right), & t \geq 0\end{cases}
$$

Here, the velocity is given by $c(x)=\frac{1}{\sqrt{\operatorname{det} H(x)}}$. The response operator $R_{w}^{T}: L_{2}(0, T ; \mathbb{C}) \mapsto L_{2}(0, T ; \mathbb{C})$ with the domain $\mathcal{D}=C_{0}^{\infty}(0, T, \mathbb{C})$ for $(26)$ is introduced as $\left(R_{w}^{T} G\right)(t):=Y_{x}^{G}(0, t)$. The eikonal function is introduced by $\tau(x):=\int_{0}^{x} \sqrt{\operatorname{det} H(s)} d s$, and $\Omega^{l}=\{x>0 \mid \tau(x)<l\}$. Then the natural setup of IP is to recover $\left.H(x)\right|_{\Omega^{T}}$ from $R_{w}^{2 T}$.

We see that the IP for the system (26), is equivalent to IP for (25). But there is one important disadvantage in studying IP for (26) which comes from (25), we need to use the specific set of controls of the type $\left(\begin{array}{c}f \\ R f\end{array}\right)$, which makes application of the BC method problematic. Instead, we will reduce (25) to Dirac-type system, and follow the scheme offered in [15].

\subsection{Dirac-type dynamical system}

We introduce the following transformation: let

$$
U=\left(\begin{array}{cc}
\cos \phi(x) & \sin \phi(x) \\
-\sin \phi(x) & \cos \phi(x)
\end{array}\right)
$$

be a unitary matrix such that $U^{*} H U=D:=\left(\begin{array}{cc}d_{1}(x) & 0 \\ 0 & d_{2}(x)\end{array}\right)$, where $d_{1}, d_{2} \geq \delta>0, d_{1}+d_{2}=1$. If $Y=U \widetilde{Y}$, then $\widetilde{Y}$ satisfies the following IBVP for Dirac-type dynamical system:

$$
\begin{cases}i D \frac{d}{d t} \tilde{Y}+J \frac{d}{d x} \tilde{Y}-\phi^{\prime}(x) \tilde{Y}=0, & x \geq 0, t \geq 0 \\ \tilde{Y}(x, 0)=0, & x \geq 0 \\ \widetilde{y}^{1}(0, t)=g(t):=\cos \phi(0) f(t)+\sin \phi(0)(R f)(t), & t \geq 0\end{cases}
$$

The response operator $R_{C D}^{T}: L_{2}(0, T) \mapsto L_{2}(0, T)$ is introduced by $\left(R_{C D}^{T} g\right)(t):=\widetilde{y}^{2}(0, t)$. We can see that $\widetilde{y}^{2}(0, t)=-\sin \phi(0) f(t)+\cos \phi(0)(R f)(t)$, so IP for (25) and for (27) are equivalent. 
Thus our first goal will be to study the dynamic IP for the following Dirac-type system:

$$
\begin{cases}i D \frac{d}{d t} V+J \frac{d}{d x} V+\psi(x) V=0, & x \geq 0, t \geq 0, \\ V(x, 0)=0, & x \geq 0, \\ v^{1}(0, t)=f(t), & t \geq 0\end{cases}
$$

where $D$ as above is a diagonal matrix with twice differentiable entries and unit trace, $\psi \in C^{2}\left(\mathbb{R}_{+}\right)$. The function $f \in \widetilde{\mathcal{F}}^{T}:=L_{2}(0, T ; \mathbb{C})$ is a boundary control. The response $R_{D}^{T}: \widetilde{\mathcal{F}}^{T} \mapsto \widetilde{\mathcal{F}}^{T}$ is introduced by $\left(R_{D}^{T} f\right)(t):=v^{2}(0, t)$. The IP consists in recovering $\left.D\right|_{\Omega^{T}},\left.\psi\right|_{\Omega^{T}}$ from $R^{2 T}$. We outline the scheme offered in $[1,15]$ :

Proposition 1. The solution to (28) admits the following representation:

$$
V(x, t)=A(x) f(t-\tau(x))+\int_{0}^{x(t)} w(x, s) f(t-\tau(s)) d s,
$$

where $\tau(s)=\int_{0}^{s} \sqrt{d_{1}(\alpha) d_{2}(\alpha)} d \alpha$ is eikonal, $x(t)$ is a function inverse to $\tau(x)$, the kernel $w=\left(\begin{array}{l}w^{1} \\ w^{2}\end{array}\right)$ is twice differentiable in $\{(x, s) \mid 0 \leq \tau(x) \leq s \leq T\}, A=\left(\begin{array}{l}a^{1} \\ a^{2}\end{array}\right)$, where $a^{1,2}$ are solutions to the following system:

$$
\begin{aligned}
i \sqrt{d_{1}} a_{x}^{1} & =\sqrt{d_{2}} a_{x}^{2}, \\
\sqrt{d_{2}}\left(\psi a^{1}+a_{x}^{2}\right) & =i \sqrt{d_{1}}\left(\psi a^{2}-a_{x}^{1}\right) .
\end{aligned}
$$

We introduce the outer space, the space o states of $(28): \mathcal{H}^{T}:=L_{2}(0, \tau(T) ; \mathbb{C})$ and a control operator $\widetilde{W}^{T}: \widetilde{\mathcal{F}}^{T} \mapsto \mathcal{H}^{T}$ acting by the rule:

$$
\left(\widetilde{W}^{T} f\right)(x):=V^{f}(x, T)
$$

The Proposition 1 implies that $\widetilde{W}^{T}$ is not an isomorphism, and the system (28) is not boundary controllable. To restore the controllability, we introduce the auxiliary system:

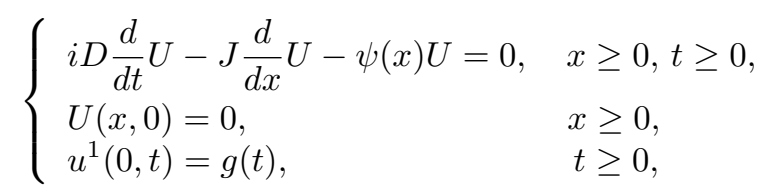

and note that solutions to (28) and (29) are connected by the formula $V^{f}=\overline{U^{\bar{f}}}$. The extended outer space is defined by $\mathcal{F}^{T}:=L_{2}\left(0, T ; \mathbb{C}^{2}\right)$, and the extended control operator $W^{T}: \mathcal{F}^{T} \mapsto \mathcal{H}^{T}$ is introduced by:

$$
W^{T}\left(\begin{array}{l}
f \\
g
\end{array}\right):=V^{f}(x, T)+U^{g}(x, T) .
$$

Proposition 2. The extended control operator is an isomorphism between $\mathcal{F}^{T}$ and $\mathcal{H}^{T}$.

The set $\mathcal{U}^{T}:=W^{T} \mathcal{F}^{T}$ is called extended reachable set. The Proposition 2 says that $\mathcal{U}^{T}=\mathcal{H}^{T}$.

We consider the operator of the Dirac-type system on a half-line: let $\mathbf{D}:=D^{-1} J \frac{d}{d x}+D^{-1} \psi$ on $L_{2}\left(\mathbb{R}_{+}, \mathbb{C}^{2}\right) \ni$ $\Phi=\left(\begin{array}{l}\Phi_{1} \\ \Phi_{2}\end{array}\right)$ with a Dirichlet condition $\Phi_{1}(0)=0$. Denote by $\theta(x, z)=\left(\begin{array}{l}\theta_{1} \\ \theta_{2}\end{array}\right)$ a solution to the following Cauchy problem for $z \in \mathbb{C}$ :

$$
\left\{\begin{array}{l}
J \theta_{x}+V \theta=z D \theta, \quad x>0 \\
\theta_{1}(0, z)=0, \quad \theta_{2}(0, z)=1
\end{array}\right.
$$


Let $d \rho$ be a spectral measure of $\mathbf{D}$, and $F: L_{2}\left(\mathbb{R}_{+} ; \mathbb{C}^{2}\right) \mapsto L_{2, \rho}\left(\mathbb{R}_{+}\right)$be the corresponding Fourier transform:

$$
\begin{gathered}
\left(F\left(\begin{array}{l}
f_{1} \\
f_{2}
\end{array}\right)\right)(\lambda)=F(\lambda)=\int_{0}^{\infty}\left(f_{1}(x) \theta_{1}(x, \lambda)+f_{2}(x) \theta_{2}(x, \lambda)\right) d x \\
f_{1}(x)=\int_{-\infty}^{\infty} F(\lambda) \theta_{1}(x, \lambda) d \rho(\lambda), \quad f_{2}(x)=\int_{-\infty}^{\infty} F(\lambda) \theta_{2}(x, \lambda) d \rho(\lambda), \\
\int_{0}^{\infty}\left(f_{1}^{2}(x)+f_{2}^{2}(x)\right) d x=\int_{-\infty}^{\infty} F^{2}(\lambda) d \rho(\lambda) .
\end{gathered}
$$

We introduce the extending connecting operator $C^{T}: \mathcal{F}^{T} \mapsto \mathcal{F}^{T}$ by the quadratic form:

$$
\left(C^{T}\left(\begin{array}{l}
f_{1} \\
g_{1}
\end{array}\right),\left(\begin{array}{l}
f_{2} \\
g_{2}
\end{array}\right)\right)_{\mathcal{F}^{T}}=\left(W^{T}\left(\begin{array}{l}
f_{1} \\
g_{1}
\end{array}\right), W^{T}\left(\begin{array}{l}
f_{2} \\
g_{2}
\end{array}\right)\right)_{\mathcal{H}^{T}}, \quad C^{T}=\left(W^{T}\right)^{*} W^{T} .
$$

The important fact in the $\mathrm{BC}$ method is that:

Proposition 3. The extending connecting operator is a positive isomorphism in $\mathcal{F}^{T}$, it admits the representation in terms of dynamic inverse data $R^{2 T}$, and spectral inverse data $d \rho(\lambda)$.

We introduce the linear manifold of Fourier images of extended states (Fourier image of extended reachable set) at time $t=T$ :

$$
B_{D}^{T}:=\left\{K(\lambda) \mid K(\lambda)=\left(F W^{T}\left(\begin{array}{l}
k_{1} \\
k_{2}
\end{array}\right)\right)(\lambda),\left(\begin{array}{l}
k_{1} \\
k_{2}
\end{array}\right) \in \mathcal{F}^{T}\right\}=F \mathcal{U}^{T}
$$

Equipped with the scalar product, generated by $C^{T}$ :

$$
[F, G]_{B_{D}^{T}}:=\left(C^{T}\left(\begin{array}{l}
f_{1} \\
f_{2}
\end{array}\right),\left(\begin{array}{l}
g_{1} \\
g_{2}
\end{array}\right)\right)_{\mathcal{F}^{T}}, \quad F, G \in B_{D}^{T}
$$

this linear space becomes a Hilbert space of analytic functions. It is also possible to define a reproducing kernel in this space (it is given in terms of a solution to a Krein equation), which makes $B_{D}^{T}$ a de Branges space. Solution of dynamic and spectral IPs for (28) and construction of corresponding de Branges space will be the subject of forthcoming publications.

\subsection{Dynamic approach to de Branges spaces}

Based on the arguments from the previous subsection, we can formulate the hypothesis about de Branges space for canonical system (25) with general Hamiltonian. First, we introduce the auxiliary system:

$$
\begin{cases}i H \frac{d}{d t} Z+J \frac{d}{d x} Z=0, & x \geq 0, t \geq 0, \\ Z(x, 0)=0, & x \geq 0, \\ z^{1}(0, t)=g(t), & t \geq 0 .\end{cases}
$$

The extending control operator $W^{T}: \mathcal{F}^{T} \mapsto \mathcal{F}^{T}$ acting in extended control space $\mathcal{F}^{T}:=L_{2}\left(0, T ; \mathbb{C}^{2}\right)$ is defined by $W^{T}\left(\begin{array}{l}f \\ g\end{array}\right):=Y^{f}(x, T)+Z^{g}(x, T)$. The extending connecting operator $C^{T}$ is given by analog to (31). Then, the de Branges space corresponding to (25) is a Fourier image of extended reachable set, equipped with a scalar product, generated by $C^{T}$.

We note that the construction of de Branges space by dynamic methods for general Hamiltonian in fact is equivalent to solving the dynamic IP for system (25) with general $H$. We note that the in studying the IP in this case, one inevitably face with two obstacles: the smoothness of $H$, and changing the rank of $H$, which reflects in the lack of the boundary controllability of the dynamical system. The authors suggest that studying the inverse dynamic problem for a Krein string $[10,19]$ will be instructive and can help to overcome difficulties connected with general Hamiltonian. 


\section{Acknowledgements}

The research of Victor Mikhaylov was supported in part by RFBR 17-01-00529-A. Alexandr Mikhaylov was supported by RFBR 17-01-00099-A; A. S. Mikhaylov and V. S. Mikhaylov were partly supported by RFBR 1801-00269-A and VW Foundation program "Modeling, Analysis, and Approximation Theory toward application in tomography and inverse problems". The authors are deeply indebted to Prof. R. V. Romanov and Prof. M. I. Belishev for valuable discussions.

\section{References}

[1] Mikhaylov A.S., Mikhaylov V.S. The Boundary Control method and de Branges spaces. Schrödinger equation, Dirac system and Discrete Schrödinger operator. Journal of Mathematical Analysis and Applications, 2018, 460 (2), P. 927-953.

[2] Remling C. Schrödinger operators and de Branges spaces. J. Funct. Anal., 2002, 196 (2), P. $323-394$.

[3] Romanov R.V. Canonical systems and de Branges spaces. URL: http://arxiv.org/abs/1408.6022, 2014.

[4] Belishev M.I. An approach to multidimensional inverse problems for the wave equation. Soviet Math. Dokl., 1988,36 (3), P. $481-484$.

[5] Belishev M.I. Boundary control in reconstruction of manifolds and metrics (the BC method). Inverse Problems, 1997,13 (5), R1-R45.

[6] Avdonin S.A., Lenhart S., Protopopescu V. Determining the potential in the Schrödinger equation from the Dirichlet to Neumann map by the boundary control method. J. Inverse Ill-Posed Probl., 2005, 13, P. 317-330.

[7] Belishev M.I. Recent progress in the boundary control method. Inverse Problems, 2007, 23 (5), R1-R67.

[8] Belishev M.I. Boundary control and tomography of Riemannian manifolds (the BC-method). Uspekhi Matem. Nauk, 2017, 72 (4), P. 3-66 (in Russian).

[9] de Branges L. Hilbert space of entire functions. Prentice-Hall, NJ, 1968.

[10] Dym H., McKean H.P. Gaussian processes, function theory, and the inverse spectral problem. Academic Press, New York etc., 1976.

[11] Avdonin S.A., Mikhaylov V.S. The boundary control approach to inverse spectral theory. Inverse Problems, 2010,26 (4), 045009 (19 pp).

[12] Belishev M.I., Mikhaylov V.S. Unified approach to classical equations of inverse problem theory. Journal of Inverse and Ill-Posed Problems, 2012, 20 (4), P. 461-488.

[13] Mikhaylov A.S., Mikhaylov V.S. Relationship between different types of inverse data for the one-dimensional Schrödinger operator on a half-line. Zapiski Nauchnykh Seminarov POMI, 2016, 451, P. 134-155.

[14] Belishev M.I. Boundary control and inverse problems: a one-dimensional version of the boundary control method. J. Math. Sci. (N. Y.), 2008, 155 (3), P. 343-378.

[15] Belishev M.I., Mikhaylov V.S. Inverse problem for one-dimensional dynamical Dirac system (BC-method). Inverse Problems, 2010, 26 (4), 045009 (19 pp)

[16] Teschl G. Jacobi operators and completely integrable nonlinear lattices. Mathematical Surveys and Monographs, 72, American Mathematical Society, Providence, RI, 2000.

[17] Mikhaylov A.S., Mikhaylov V.S. Dynamical inverse problem for the discrete Schrödinger operator. Nanosystems: Physics, Chemistry, Mathematics, 2016, 7 (5), P. 842-854.

[18] Mikhaylov A.S., Mikhaylov V.S. Dynamic inverse problem for the Jacobi matrices. URL: https://arxiv.org/abs/1704.02481, 2017.

[19] Krein M.G. On the one method of effective solving the inverse boundary value problem. Dokl. Akad. Nauk. USSR, 1954, 94 (6), P. 987-990. 